23.5\%) Risk of recurrence prediction was quite similar between calculated and observed. $(26.7 \%$ calculated and $25.6 \%$ observed) In low risk group, FIGO stage $(p=0.004)$ and old age $(p=0.04)$ was related of the risk of recurrence using univariate analysis.

Conclusion* The KGOG-1024 risk assessment model accurately predicted a distant recurrence after chemoradiation in patients with locally advanced cervical cancer, especially in intermediate-risk group. The model may be helpful in identifying patients who may benefit from adjuvant systemic treatment after chemoradiation.

\section{ROBOTIC-ASSISTED LYMPHADENECTOMY IN PREGNANCY AS CERVICAL CANCER STAGING - A CASE REPORT}

B Bittnerová*, R Pilka, V Staňková, M Janošík, P Dzvinčuk, R Marek. University Hospital Olomouc, Department of Obstetrics and Gynaecology, Olomouc, Czech Republic

\subsection{6/ijgc-2021-ESG0.98}

Introduction/Background* Cervical cancer represents one of the most commonly diagnosed tumours in pregnancy. Roboticassisted surgery is considered the standard method for surgical staging of cervical cancer. There is a very limited data in the available literature on the use of this method to perform lymphadenectomy in patients with cervical cancer during pregnancy. In the case report we describe the procedure of robotic pelvic lymphadenectomy in a patient with cervical cancer T1b in pregnancy.

Methodology A 43 years old nulliparous woman pregnant for the third time was referred to our department with a confirmed cervical adenocarcinoma in the 15 th week of pregnancy. According to the preoperative imaging examination (pelvic MRI scan, expert sonography), she suffered from stage T1b cervical cancer. The patient preferred the continuation of the pregnancy. Informed about the associated risks, she signed an informed refusal rejecting proposed radical surgical treatment. Based on the recommendation of the multidisciplinary team of gynaecological oncology, a surgical staging procedure was indicated before possible neoadjuvant systemic therapy. The robotic-assisted laparoscopic lymphadenectomy was performed on 21.5.2020 as a surgical modality of a minimally invasive approach. The procedure was performed without any complications, blood loss was up to $20 \mathrm{ml}$. Histopathological examination excluded the cancer dissemination into the lymph nodes. Subsequently, systemic treatment (cisDDP + Paclitaxel) was administered for 9 cycles to ensure adequate foetal maturity. Planned caesarean sections combined with a radical surgical treatment - radical hysterectomy and bilateral adnexectomy - were performed in gestational age 30 weeks and 5 days.

Result(s)* According to definitive histology and after the neoadjuvant treatment the stage of the disease was classified as FIGO IA1 ypT1a1 ypN0 ypMX Mandard TRG3. A close follow-up is ongoing in the patient.

Conclusion* In pregnant patients with cervical cancer, robotassisted laparoscopic lymphadenectomy is a very gentle and effective method of staging.

\section{Diagnostics}

\section{ACCURACY OF TRANSVAGINAL/TRANSRECTAL ULTRASOUND IN PREOPERATIVE PELVIC LYMPH NODE ASSESSMENT IN CERVICAL CANCER PATIENTS}

${ }^{1} \mathrm{~F}$ Fruhauf*, ${ }^{1} \mathrm{~T}$ Ballaschova, ${ }^{2} \mathrm{M}$ Zikan, ${ }^{1} \mathrm{~L}$ Dostalek, ${ }^{3} \mathrm{P}$ Dundr, ${ }^{1} \mathrm{D}$ Cibula, ${ }^{1} \mathrm{D}$ Fischerova. 'General University Hospital, First Faculty of Medicine, Charles University, Department of Obstetrics and Gynaecology, Prague, Czech Republic; ${ }^{2}$ Bulovka University Hospital, First Faculty of Medicine, Charles University, Department of Obstetrics and Gynaecology, Prague, Czech Republic; ${ }^{3}$ General University Hospital, First Faculty of Medicine, Charles University, Institute of Pathology, Prague, Czech Republic

\subsection{6/ijgc-2021-ESG0.99}

Introduction/Background* The aim of the study was to evaluate diagnostic accuracy of ultrasound in preoperative assessment of pelvic lymph nodes (LNs) in cervical cancer patients. Methodology Patients were retrospectively included if they met following inclusion criteria: 1) histologically verified cervical cancer; 2) preoperative ultrasound examination performed by one of three experienced sonographers (transvaginal or transrectal and transabdominal approach); 3) surgical lymph node staging (sentinel lymph node biopsy, SLNB and/or systematic pelvic lymphadenectomy, PLND or pelvic lymph node debulking). The final pathological examination served as the

Abstract 133 Table 1 Characterisation of study population

\begin{tabular}{|c|c|}
\hline Characteristic & Value $(\mathrm{N}=394)^{\mathrm{a}}$ \\
\hline Age, median (range), years & $43(20-82)$ \\
\hline BMI, median (range) & $24.5(14.1-44.9)$ \\
\hline \multicolumn{2}{|l|}{ Histological type } \\
\hline Squamous cell carcinoma & $298(75.6)$ \\
\hline Adenocarcinoma & $79(20.0)$ \\
\hline Neuroendocrine carcinoma & $13(3.4)$ \\
\hline Other & $4(1.0)$ \\
\hline \multicolumn{2}{|l|}{ Stage (FIGO 2009) } \\
\hline $\mid A 1$ & $18(4.5)$ \\
\hline IA2 & $15(3.8)$ \\
\hline IB1 & $236(60.0)$ \\
\hline IB2 & $44(11.1)$ \\
\hline IIA1 & $5(1.2)$ \\
\hline IIA2 & $2(0.5)$ \\
\hline IIB & $47(12.0)$ \\
\hline IIIA & $1(0.3)$ \\
\hline IIIB & $3(0.8)$ \\
\hline IVA & $2(0.5)$ \\
\hline IVB & $21(5.3)$ \\
\hline \multicolumn{2}{|l|}{ Type of pelvic LN staging } \\
\hline SLNB only & $87(22.1)$ \\
\hline SLNB + PLND & $241(61.1)$ \\
\hline PLND only & $35(8.9)$ \\
\hline Debulking of pelvic LNs only & $31(7.9)$ \\
\hline \multicolumn{2}{|l|}{ Pelvic LN status } \\
\hline Normal & $308(78.2)$ \\
\hline Macrometastases $( \pm \mathrm{MIC} \pm \mathrm{ITC})$ & $53(13.5)$ \\
\hline Micrometastases ( \pm ITC) & $23(5.8)$ \\
\hline Isolated tumour cells only & $10(2.5)$ \\
\hline
\end{tabular}

${ }^{a}$ Data are presented as number (percentage) of patients unless otherwise indicated. 
reference standard. Lymph nodes with macrometastases (the largest diameter $>2 \mathrm{~mm}$ ) were considered positive, while LNs with isolated tumour cells (ITC) and micrometastases (MIC) were considered negative.

Result(s)* 394 patients meeting the inclusion criteria between 2009 a 2019 were enrolled into the study. The characteristics of study population are shown in table 1 . Squamous cell carcinomas were most common (298/394) and the majority of cases was represented by early stage cancers (274/394), specifically IB1 FIGO 2009 (236/394). Macrometastes in pelvic LNs were pathologically confirmed in 53 patients $(13.5 \%)$ and micrometastases solely in 23 patients (5.8\%). Ultrasound failed to detect pelvic lymph node macrometastases in 15 patients $(3.8 \%)$ and median largest diameter of these unidentified metastases was $6 \mathrm{~mm}$ (range $3-11 \mathrm{~mm}$ ). There were 27 false positive ultrasound findings (6.9\%). Ultrasound reached sensitivity $71.7 \%$, specificity $92.1 \%$, PPV 58.5\%, NPV 95.4\% and overall accuracy $89.3 \%$.

Conclusion* Transvaginal/transrectal ultrasound is a reliable method for preoperative assessment of pelvic LNs in cervical cancer patients. It showed similar accuracy in detection of nodal macrometastases as reported for other imaging modalities. Similarly to all imaging methods, it had low sensitivity in detection of small volume macrometastases and micrometastases.

Key words: cervical cancer, lymph nodes, ultrasound, diagnostic accuracy

Klikně te nebo klepně te sem a zadejte text.

\section{THE UTILITY OF BIOMARKERS FOR OVARIAN CANCER RISK ASSESSMENT IN PRIMARY CARE: A PILOT STUDY}

${ }^{1} \mathrm{C}$ Barr*, ${ }^{2} \mathrm{G}$ Funston, ${ }^{3} \mathrm{D}$ Jeevan, ${ }^{3} \mathrm{SS}$ Sundar, ${ }^{4} \mathrm{LT}$ Mounce, ${ }^{5} \mathrm{E}$ Crosbie. ${ }^{1}$ Manchester University NHS FT, Division of Gynaecology, Manchester, UK; ${ }^{2}$ University of Cambridge, Department of Public Health and Primary Care, UK; ${ }^{3}$ University of Birmingham, Institute of Cancer and Genomic Sciences, UK; ${ }^{4}$ University of Exeter, Institute of Health Research, UK; ${ }^{5}$ The University of Manchester, Division of Cancer Sciences, UK

\subsection{6/ijgc-2021-ESG0.100}

Introduction/Background* Ovarian cancer is the leading cause of mortality from gynaecological malignancy. Survival improves with early diagnosis, however, early detection in primary care is challenging. The current blood test, cancer antigen 125 (CA125), has limited sensitivity and specificity for early disease. Human Epididymis 4 (HE4) is a promising diagnostic biomarker. We aimed to investigate the diagnostic accuracy and clinical utility of serum HE4 in a symptomatic primary care population.

Methodology We conducted a prospective observational study testing HE4 on primary care serum CA125 samples from women with suspected ovarian cancer in Manchester, UK, between April 2018 and April 2019. Serum HE4 was measured using chemiluminescent enzyme immunoassays following routine CA125 testing for clinical care. HE4 thresholds of $77 \mathrm{pmol} / \mathrm{L}$ and $150 \mathrm{pmol} / \mathrm{L}$ were used. The primary outcome was final diagnosis within 12 months of testing. Clinical outcomes were collected from hospital electronic patient records. Receiver operator characteristic (ROC) curves with area under the curve (AUC), sensitivity and specificity were calculated for CA125 and HE4 both alone and in combination. Age adjusted HE4 thresholds were calculated with linear regression models.
Result(s)* 1247 patients were included, with a mean age of 50 years (SD 15.7). 100 women had epithelial ovarian cancer; including 82 invasive and 18 borderline ovarian tumours. There was little difference in overall performance of CA125 and HE4 (AUC 0.932 vs 0.914 respectively). At a threshold of $77 \mathrm{pmol} / \mathrm{L}$, HE4 alone had a better sensitivity than CA125 [89\% (95\%CI 81.2-94.4) vs $81 \%(95 \%$ CI 71.9-88.2)] but a worse specificity $[75.6 \% \quad(95 \% \mathrm{CI} \quad 73-78)$ vs $92.2 \% \quad(95 \% \mathrm{CI}$ 90.4-93.6)]. HE4 and CA125 combined had improved sensitivity compared with CA125 alone (93\%, 95\%CI 86.1-97.1), but at a significant cost to specificity $(70 \%, 95 \% \mathrm{CI}$ 67.3-72.6). Serum HE4 levels were correlated with increasing age $(p$ $<0.001)$ and worsening eGFR $(\mathrm{p}<0.001)$. Age adjusted HE4 cut-offs marginally improved the specificity of CA125, however the numbers were small per age category and require validation in larger cohorts.

Conclusion* HE4 adds little to current diagnostic pathways in primary care. Age-adjusted thresholds may improve accuracy, but not sufficiently to recommend routine use at present.

\section{MYOMETRIAL INFILTRATION ASSESSMENT IN LOW-RISK ENDOMETRIAL CANCER BY 3D TRANSVAGINAL ULTRASOUND AND DIFFUSION-WEIGHTED MAGNETIC RESONANCE IMAGING}

${ }^{1} \mathrm{~N}$ Carreras Diéguez*, ${ }^{2} \mathrm{M}$ Munmany, ${ }^{3} \mathrm{C}$ De Guirior, ${ }^{1} \mathrm{P}$ Fusté, ${ }^{3} \mathrm{I}$ Matas, ${ }^{1} \mathrm{~B}$ Diaz-Feijoo, ${ }^{1} \mathrm{AG}$ Glickman, ${ }^{4} \mathrm{~L}$ Buñesch, ${ }^{1} \mathrm{~N}$ Agustí, ${ }^{3} \mathrm{C}$ Ros, ${ }^{4} \mathrm{M}$ Sebastià, ${ }^{1} \mathrm{M}$ Del Pino, ${ }^{5} \mathrm{~A}$ Saco, ${ }^{4} \mathrm{C}$ Nicolau, ${ }^{5} \mathrm{~J}$ Ordi, ${ }^{1} \mathrm{~A}$ Torne. ${ }^{1} \mathrm{Hospital}$ Clínic de Barcelona, Gynaecologic Oncology Unit, Barcelona, Spain; ${ }^{2}$ Hospital Clínic de Barcelona, Gynaecology, Obstetrics and Neonatology, Barcelona, Spain; ${ }^{3}$ Hospital Clínic de Barcelona, Gynaecology, Obstetrics and Neonatology, Barcelona, Spain; ${ }^{4}$ Hospital Clínic de Barcelona, Radiology, Barcelona, Spain; ${ }^{5}$ Hospital Clínic de Barcelona, Pathology Department, Barcelona, Spain

\subsection{6/ijgc-2021-ESG0.101}

Introduction/Background* In patients with early-stage, grade 12, endometrioid endometrial cancer, preoperative assessment of myometrial invasion is essential to define the need of pelvic and paraaortic lymph node dissection. Our aim was to evaluate the role of three-dimensional transvaginal ultrasound (3D-TVUS) and diffusion-weighted magnetic resonance imaging (DW-RMI) for the assessment of myometrial infiltration in patients with well-differentiated (G1) or moderately differentiated (G2) endometrioid endometrial carcinoma (EC).

Methodology We performed a retrospective observational study. Myometrial infiltration was assessed by 3D-TVUS and DW-MRI in 152 women with G1 or G2 endometroid EC who underwent surgical treatment in a tertiary referral center between 2012 and 2019. Sensitivity, specificity, predictive values and accuracy for the two techniques and for a combination of both were computed. Definitive histopathological data in the surgical specimen regarding myometrial infiltration was used as 'Gold Standard'.

Result(s)* One hundred and fifty-two patients were included, $120(79 \%)$ patients presented myometrial infiltration $<50 \%$ in postoperative analysis of surgical specimen and $32(21 \%)$ patients presented deep myometrial infiltration (>50\%). 3DTVUS and DW-MRI showed an agreement of $78.9 \%$ with a kappa index of 0.44 for the detection of deep myometrial infiltration. Sensitivity, specificity and accuracy of 3D-TVUS for the detection of deep myometrial infiltration were $71.0 \%$, $80.5 \%$ and $78.5 \%$ respectively. Evaluation of myometrial 